\title{
VIOLATIONS OF THE LAW OF THE EXCLUDED MIDDLE IN MEDIA DISCOURSE
}

\author{
Ramis R. Gazizov ${ }^{1}$ \\ Olesya A. Vol'f ${ }^{2}$
}

\begin{abstract}
The paper is devoted to the study of violations of one of the laws of formal logic: the law of the excluded middle; those violations are found in modern media discourse. The authors examined the relationship between deviations from the law of noncontradiction (contradiction) and the law of the excluded middle and came to the conclusion that violations of the law of the excluded middle occur in speech only in symbiosis with violations of the law of non-contradiction. The analysis of examples from journalistic tests showed that deviations from the law of the excluded middle can be of two types depending on the reasons for their occurrence. If a logical rule is violated unknowingly, a logical error appears in the text. In the case when the author intentionally goes to damage the logical structure of the utterance, the violation is
\end{abstract}

a technique. In turn, the goals of the addressee predetermine the division of techniques into manipulative (in the case when a recipient should not notice logical inconsistencies) and stylistic ones: tropes and figures (which are designed to influence an addressee openly).

Keywords: media discourse, journalistic text, the law of non-contradiction (contradiction), the law of the excluded middle, illogism, mistake, manipulation, stylistic means, alogism.

\section{Introduction}

Today in conditions of the avalanche increasing of information volumes that daily falls upon a person issues of informational ecology and security are becoming extremely urgent. Important actors in the global

\footnotetext{
${ }^{1}$ Kazan Federal University, Kremlyovskaya str., 18, 420008, Kazan, Russian Federation, gazizov-da@yandex.ru. (919) 684-00-77

2 Katanov Khakass State University, Lenina str., 90, 655000, Abakan, Russian Federation, oles-sya@mail.ru. (913) 443-17-79
} 
communication process are journalists: employees of traditional media and multimedia editorial staff. Sociologists who recently spoke about the reluctance of an Internet person to read long texts and predicted quick death for analytical journalism note resurgence in the interest of the mass audience in high-quality journalistic materials. This, for example, is stated in a study on the development of the journalistic investigative genre in Russia [1]. People again want to read long-reads and receive objective, logically verified, reliable information. As a result, the scientific interest in factors influencing the development (progress or degradation) of journalism has increased.

The requirement to observe the rules of logic when creating text is, in our opinion, one of the main ones in the profession of a journalist. The ability to think correctly and, further, correctly convey your thoughts means that the journalist has the most important professional qualities: the ability to analytical thinking and the desire for an objective transfer of information. The reliability of the facts and opinions presented in journalistic materials directly depends on the author following logical laws.

Traditionally, four laws of formal logic occupy a special place in logic, which most researchers consider the main: the law of identity, the law of non-contradiction (contradiction), the law of the excluded middle and the law of sufficient reason.

In this paper, we turn to violations of the law of the excluded middle in journalistic texts.

\section{Methods}

The purpose of this work is to determine the linguistic status and features of the realization of conscious and unconscious deviations from the law of the excluded middle in the media discourse. The methodological basis of the study was the principles of systemic, structural and integrated analysis. The authors used systematization and various methods of analysis (interpretation, classification, pragmatic and contextual analysis, as well as content analysis) as methods. Speech examples in the amount of 215 units are taken from the materials of the Russian media and communication. 


\section{Sicieite}

Periódico do Núcleo de Estudos e Pesquisas sobre Gênero e Direito

Centro de Ciências Jurídicas - Universidade Federal da Paraíba

V. 8 - No 06 - Ano 2019 - Special Edition

ISSN | 2179-7137 | http://periodicos.ufpb.br/ojs2/index.php/ged/index

\section{Results And Discussion}

First of all, it is necessary to differentiate two logical laws that are close to each other in their content: the law of non-contradiction and the law of the excluded middle.

In the fourth book of Metaphysics, Aristotle gives the essence of the law of non-contradiction: "it is impossible that the same thing at the same time should be and should not be inherent in the same thing in the same respect" [2, p. 125].

The law of the excluded middle in the wording of Aristotle reads as follows: "... nothing can be in the middle between two conflicting judgments about one; each separate predicate must either be affirmed or denied" [2, p. 141]. Some researchers, for example, Warayuth Sriwarakuel, consider the law of the excluded middle to be the main of Aristotelian laws of formal logic: "Which is the most fundamental among these three laws? I believe that it is the law of the excluded middle. Why so? It is because we can convert the other two laws into the law of the excluded middle." [3, p. 124]

Most clearly and reasonably, in our opinion, the differentiation of the phenomena studied is presented in the work of Y. A. Slinin [4]. This author says that for understanding the difference between the concepts to which the two mentioned laws apply, the terms "opposite" and "contradiction" exist.

To interpret the law of noncontradiction, both characteristics can be used, i.e. the law applies to both counter and contradictive logical relationships between concepts. For example, the judgments "A man is tall" and "A man is short" can be simultaneously false (if the person is of average height), but they cannot be true at the same time. In this case, the adjectives "high" and "low" are in a contra-relationship: they are located at different ends of the scale indicating the growth of a person, and there are other links between them, of course ("not very low", " medium height", " slightly higher than average", etc.). The concept of "contradiction" can be illustrated by the following example: the statements "The man is alive" and "The man is dead" cannot be true at the same time, nor can they be false at the same time. There can be no middle link between two members of the counter-narrative opposition. 
As for the law of the excluded middle, it applies only to conflicting concepts that are in counter-narrative relationships (as in the last example).

Since the law of noncontradiction extends in some cases to the same type of statements, to which the law of the excluded middle applies, the question arises of how speech examples containing violations of these laws relate, and whether their differentiation is possible.

When studying the role of logical laws in the creation of the funny, a researcher N.V. Safonova conducted a study of 500 jokes in which she did not find a single case where the "pure" violation of the law of the excluded middle would serve as the mechanism for the formation of a funny matter. The author came to the conclusion that "there are no funny situations where only the law of the excluded middle is violated and the law of non-contradiction could not be applied" [5, p.271].

Based on the results obtained by N.V. Safonova, we suggested that in other statements, built on the violation of the law of the excluded middle, but not creating a comic effect, this law will be contaminated with the law of non- contradiction. This hypothesis was confirmed by the examples we discovered.

At the same time, complete identification of deviations from the law of non-contradiction and the law of the excluded middle, in our opinion, is not advisable, since in case of combining the opposite concepts in one context only the law of non-contradiction is violated. See an example: "There are really few fools, but they are so cleverly placed that we permanently stumble upon them" (05/27/18, http://bibo.kz/kipa/343204durakov-na-samom-dele-nemnogonooni-tak-lovko.html). The joke is based on a combination of opposing concepts of "few" and "permanently", between which other options are possible (for example, "often"), therefore the law of the excluded middle is not applicable to them.

Therefore, violations of the law of the excluded middle occur in speech practice only "in the concert with" the law of non-contradiction.

In linguistics, deviations from logical laws can have a different status depending on the reasons for their occurrence. If a logical rule is violated unknowingly, due to inattention or 
inability to correctly perform logical operations, a logical error appears in the text. Otherwise, when the author intentionally goes to damage the logical structure of the utterance, such a retreat is considered to be a technique. In turn, the goals of an addressee predetermine the division of techniques into manipulative (in the case when a recipient should not notice a logical "catch") and stylistic ones - tropes and figures (which are designed to influence the addressee openly). A researcher E.E. Yermakovich, who proposed differentiating conscious logical deviations ("paralogisms") into manipulative (sophisms, tricks) and stylistic ones, based on the category of "distortion - undistortedness of transmitted information" [6, p. 3].

Consider these types of violations concerning the law of the excluded middle in journalistic texts.

Inadvertent violations, or

errors. Logical errors in the media more than once became the object of study. However, in scientific works on this topic, there is almost no mention of the law of the excluded middle in general and of specific mechanisms for making such errors in particular. We will try to fill this gap.

When monitoring the media, we found the following types of violations concerning the law of the excluded middle, which we have considered it appropriate to typologise in accordance with the tier of the language structure in which they are implemented.

1) Derogations from the law of the excluded middle at the textual level.

The logical organization of the text implies compliance with the rules for constructing the composition of the speech work and its parts, as well as the presence of subject-semantic relationships between these parts. In journalistic materials, there are violations of the law of the excluded middle, when something is affirmed in one part of the text and the same is denied in the other. We illustrate what was said by the example of the article "Winter Is Coming" from the newspaper "Shans":

"SHANS" was not used to pouring baseless facts unconfirmed with documents, so we made a request to the administration. Its employees did not deny us (for which we thank them very much) and, within the time period 
established by law, sent a copy of the protocol certified by seals and signatures concerning the meeting of parishioners of the Peter and Paul Church. <...>

$$
\text { After "SHANS" began its }
$$
investigation in the Karatuz parish, many Internet users were quick to accuse us of biased coverage of the situation, saying that we are not showing the other side of the conflict. Yes, we would be happy! But how to show it, dear, if this second side does not make contact? <..> The district administration completely went into the wilderness defence $(06 / 01 / 2018$ http://shans.online/zima-blizko/).

The author of the article, accusing the administration of the Karatuzsky district of the Krasnoyarsk Territory of the reluctance to contribute to the journalistic investigation at the end of the article, as if he had forgotten that he had previously expressed gratitude to the workers of the same structure for the prompt provision of information. The judgments "Administration helps" and "Administration does not help" are simultaneously affirmed as true, which is impossible if we observe the logical law that we are studying.
2) Violations of the law of the excluded middle on the syntactic tier.

The contradictions at the level of sentences and phrases are manifested in a deviation from the rules for constructing the indicated language units, and in violations of syntactic relations. See: Users are offered four options for the answer, each of which is stranger than the other $(06 / 01 / 2018$, http://shans.online/eto-fiasko-bratany/).

In the statement, the judgments clash: "Each of the four options is stranger than the others" and "If one of the options is stranger, then the rest should be less strange". The journalist could not correctly construct a stable phrase ("one is stranger than the other"), which caused a logical error.

3) Errors associated with deviations from the law of the excluded middle at the lexical level.

Logical errors often arise due to violations of the lexical-semantic valence or confusion in a speech of words related by paradigmatic relationships (synonyms, antonyms, homonyms, etc.). Deviations from the law of the excluded middle include the levelling of antonyms and the mixing of 
enantiosemants. We illustrate the above quotes from journalistic articles:

(1) Initially, the Varlamovs were not put on the line as needing housing.

- Because they need at least 7 squares per person, and we have 23, says Elena $(06 / 01 / 2018$ http://shans.online/a-kuda-uzhe-pisat/).

The paper explains that 3 people live in a room on 23 square meters, therefore, about 7.6 squares per one family member. And in order to queue for housing, there should be no more than 7 square meters per person. In this example, instead of the word "maximum", its antonym is used, which makes the statement illogical.

(2) As follows from the same petition of the parishioners of the Peter and Paul Church to Patriarch Cyril and Metropolitan Panteleimon, in winter the temperature in the church did not rise above 8-10 Celsius degrees. The congregation fell ill, and those who worked in the church did not have time to leave for sick leaves (06/01/2018, http://shans.online/zima-blizko/).

In the second sentence, there is an internal contradiction caused by the contextual enantiosemia of the expression "did not have time". On the one hand, the information presented in the text can be interpreted as the fact that church workers did not have time to leave for sick leave due to work. On the other hand, the context suggests that the author had otherwise in mind: the employees did not have time to leave one sick leave, as they had to take another.

In all the language tiers examined, inadvertent violations of the law of the excluded middle arise due to the weakness of the author of the texts in the norms of the Russian literary language and ignorance of the rules of logic.

Deliberate violations as manipulative methods. Various aspects of the manipulation by public consciousness (mind control) through the media have been studied for quite some time; see, for example, the works by G.A. Gladney, M.C. Ehrlich [7], R.R. Gazizov and T.A. Nagovitsyna [8], and many others. When analysing manipulative technologies, deviations from the laws of formal logic are usually mentioned. Here is an excerpt from an article in the newspaper "Khakassia", in which violation of the law of the excluded middle is a manipulative trick: 
"Today in Chernogorsk, the second meeting of the internal party vote participants was held to determine the candidatures for the subsequent nomination from the United Russia party in the election of the head of the republic. $\langle\ldots\rangle$

Each of the participants began with a short story about themselves.

The first with his program was Oleg Gavlovsky. Its basis is the socioeconomic development of the republic, and ways of filling the republican budget.

Victor Zimin came next. His report was voluminous for the reason that he reported to the party members on the activities of the republican government over the past nine years of work, and threw a bridge from concrete examples into the future: what else needs to be done. He stressed that the implementation of existing programs will continue” (10.06.18, http://gazeta19.ru/index.php/vkhakasii/item/12162-v-chernogorskevstretilis-uchastniki-vnutripartijnogogolosovaniya-edinoj-rossii).

The opinions expressed in the presented text cannot be both true or false at the same time: "Each of the participants briefly spoke about himself" and "The report of one of the participants was not short." The journalist uses the contradiction to convey to his reader the idea that the two prospective candidates for the post of the head of the Republic of Khakassia have practically nothing to tell about themselves compared to the third candidate, the current head of the Republic. Violation of the law of the excluded middle is implemented at the textual level, updated not only by the use of contradictory lexical units in different parts of the paper, but also by the volume of the text devoted to one or another candidate: one paragraph of two lines were assigned to Oleg Gavlonsky, two paragraphs of eight lines to Valentina Filimonova, and ten paragraphs of the text (24 lines) were written about Viktor Zimin. In our opinion, the author deliberately resorted to an illogical composition containing parts that are inconsistent with each other in terms of information volume, in order to covertly influence the consciousness of his audience, forcing readers to believe in the advantages of a particular candidate.

Deliberate violations as stylistic means. As researchers I.V. 
Pekarskaya and A.Yu. Spalevich note, "Questions of speech influence become of great importance when communicants interact through literary and journalistic texts" [9, p.87]. Obviously, one of the most effective tools for verbal influence in a journalistic text is stylistic means: tropes and figures, which "are amplifiers of the figurativeness of speech" $[10$, p.106-195].

Some of the stylistic tools are based on a deliberate and expedient deviation from logical rules, including the law of the excluded middle. See an example:

So, it turns out that a feldshermidwife station in Berenzhak is sheer profanity and window dressing. It seems to be there, but in fact, it is not $(01 / 06 / 2018$, http://shans.online/tserkov-kgb-isvyazi/).

The journalist uses a paradox: a judgment that has at first glance a contradictory form, which in fact is a deeper meaning of the statement. In this case, a contradictory scheme is implemented at the sentence level (see H. Field in more detail about the formal schemes of truth in a formal syntactic theory [11]).
Following the concept by the head of the Abakan scientific school I.V. Pekarskaya, we believe that the basis for the construction of stylistic means is formed by certain principles: paradigmatic and syntagmatic, general and particular [10, p. 176-190]. One of the principles is alogism which implies a conscious and effective deviation in a speech from the formal logic laws [12, p.323]. Exploring the specific mechanisms of the implementation of alogisms, O.A. Wolf includes in the set of eloquatives based on deviations from the law of the excluded middle, such expressive means as a paradox, absurdity, apophasis, paralepsis, etc. [13, p. 37-41]. In our opinion, it is advisable to carry out the study of stylistic tools built on the principle of alogism, based on several criteria (structural, semantic, functional) within the framework of field theory. In this regard, a full description of the eloquatives based on deviations from the law of the excluded middle will be the subject of a separate paper and will ultimately provide a systematic description of all stylistic tools based on an intentional violation of logical laws.

\section{Summary}


The study conducted by the authors and devoted to violations of the law of the excluded middle in the modern media discourse made it possible to draw the following conclusions:

1. Violations of the law of the excluded middle are observed only in cases of conflicting concepts that are in counter-narrative relations. In contrast, deviations from the law of noncontradiction cover both the counter (opposite) and the counter-narrative (contradictory) logical relations between the concepts.

2. In the statements built on violation of the law of the excluded middle, this law is contaminated with the law of non-contradiction. However, the full identification of deviations from the law of non-contradiction and the law of the excluded middle is not advisable, since, in the case of combining in the same context of opposite concepts, only the violation of the law of noncontradiction is observed.

3. Deviations from the law of the excluded middle can be of two types depending on the reasons for their occurrence. If a logical rule is violated unknowingly, a logical error appears in the text. In the case when the author intentionally goes to damage the logical structure of his utterance, the violation is a technique. In turn, the goals of the addressee predetermine the division of techniques into manipulative (in the case when a recipient should not notice logical inconsistencies) and stylistic ones - tropes and figures (which are designed to influence the addressee openly).

\section{Conclusions}

This study examined the features inherent in violations of the law of the excluded middle in modern media. Correlation of illogical speech utterances found in journalistic texts showed that deviations from the law of the excluded middle are at the same time violations of the law of non-contradiction. The analysis of illustrative material allowed the authors to argue that the studied alogisms operate in a media discourse in three ways: inadvertent deviations from the logical law (errors), deliberate deviations with the aim of deceiving or confusing the addressee (manipulative techniques), and deliberate deviations made by the authors intentionally to enhance the pragmatic effect (stylistic means - tropes and figures). 


\section{Acknowledgements}

The work is performed according to the Russian Government Program of Competitive Growth of Kazan Federal University.

\section{References}

T.A. Nagovitsyna, R.R. Gazizov, "Trends of Journalistic Investigation Genre Development in Russian Media”, The Social Sciences (Pakistan), Vol. 10, No. 7, pp. 1795-1797, 2015.

Aristotle, "Metaphysics", Works: in 4 volumes, Vol. 1, Moscow: Mysl, 550 p., 1976.

W. Sriwarakuel, "The Hartshornian Way: a Way to Solve Dualism", Bulletin of the Peoples' Friendship University of Russia. Series: Philosophy, No. 4, pp. 122-130. 2011.

Ya.A. Slinin, "The Leibniz principle of contradiction", Logical and philosophical studies, Volume 13, No. 3, P. 57-76, 2016.

N.V. Safonova, "The Role of the Law of the excluded middle in the Formation of the Ridiculous", Scientific notes of Taurida National University named after V. I. Vernadsky. Series "Philosophy. Culturology. Political science. Sociology", Volume 23 (62), No. 2, pp. 267-272, 2010.

E.E. Ermakovich, "Logical errors and paralogical techniques of manipulation in the media (based on the texts of the news portal LENTA.RU)", Ecology of language and communicative practice, No. 1, P. 1-9, 2016.

G.A Gladney, M.C. Ehrlich, "CrossMedia Response to Digital Manipulation of Still and Moving Images," Journal of Broadcasting and Electronic Media, Vol. 40, No. 4, pp.496-508, 1996.

R.R. Gazizov, T.A. Nagovitsyna, "Features of Manipulative Technologies in Russian Political Discourse (on the Example of the Tatarstan Republic Mass media)", Journal of Organizational Culture, Communications and Conflict, Vol. 20, № Special Issue 2, pp. 14-18, 2016.

I.V. Pekarskaya, A.Yu. Spalevich, "The Elocative Impact of a Journalistic Text: 
From the Type of the Author's Linguistic

Personality to the Selection of Language

Expression Means," Ecology of

Language and Communicative Practice,

No. 4, pp. 86-96, 2017.

I.V. Pekarskaya, Blending in the context of the problem concerning systemic stylistic resources of the Russian language, Part 1, Abakan: Publishing House of KSU named after N.F. Katanov, 248 p. 2000.

H. Field, "Saving the Truth Schema from Paradox", Journal of Philosophical Logic, Vol. 31, No. 1, pp. 1-27, 2002.

O.A. Wolf, "Mechanisms for the formation of stylistic figures of illogism using a deliberate violation of the law of sufficient reason”, Philological sciences. Issues of Theory and Practice, No. 4 (82), Part 2, P. 323-327, 2018.

O.A. Wolf, "Figures built on the principle of alogism: the problem of nomenclature description", Bulletin of the Khakass State University named after N.F. Katanov. Issue 22, pp. 37-41, 2017 